\title{
Al based Computational Trust Model for Intelligent Virtual Assistant
}

\author{
Babu Kumar* \\ AIIT, Amity University Uttar Pradesh, Noida, India \\ kbabu551@gmail.com \\ Ajay Vikram Singh \\ AIIT, Amity University Uttar Pradesh, Noida, India \\ avsingh1@ amity.edu \\ Parul Agarwal \\ Jamia Hamdard University, New Delhi, India \\ pagarwal@jamiahamdard.ac.in
}

Received: 24/July/2020 Revised: 14/Nov/2020 Accepted: 09/Jan/2021

\begin{abstract}
The Intelligent virtual assistant (IVA) also called AI assistant or digital assistant is software developed as a product by organizations like Google, Apple, Microsoft and Amazon. Virtual assistant based on Artificial Intelligence which works and processes on natural language commands given by humans. It helps the user to work more efficiently and also saves time. It is human friendly as it works on natural language commands given by humans. Voice-controlled Intelligent Virtual Assistants (IVAs) have seen gigantic development as of late on cell phones and as independent gadgets in individuals' homes. The intelligent virtual assistant is very useful for illiterate and visually impaired people around the world. While research has analyzed the expected advantages and downsides of these gadgets for IVA clients, barely any investigations have exactly assessed the need of security and trust as a singular choice to use IVAs. In this proposed work, different IPA users and non-users $(\mathrm{N}=1000)$ are surveyed to understand and analyze the barriers and motivations to adopting IPAs and how users are concerned about data privacy and trust with respect to organizational compliances and social contract related to IPA data and how these concerns have affected the acceptance and use of IPAs. We have used Naïve Byes Classifier to compute trust in IVA devices and further evaluate probability of using different trusted IVA devices.
\end{abstract}

Keywords: Artificial Intelligence; Virtual Assistant System; Product Quality; Trust, Privacy; Security; Voice Recognition; Naïve Byes Classifier.

\section{1- Introduction}

IVA is like an assistant for its users which completes the task when it gets the command. The best feature of these virtual assistant applications is that it gets active only when it hears a keyword like 'Alexa' or 'okay Google'. IVAs are AI based and able to learn from the commands and the human behavior so that next time it could easily understand what the user's command all is about. An IVA also reduces the human effort for example now no user has to search any contact to call to any person, they just have to speak the 'call' with the user contact and instead of going to a website and searching about the weather of your city you simply have to speak 'what is the weather today or in your language' and you will get quickly get the weather status of your city. The assistant application first records your voice and saves using speech recognition algorithm and it directly sends all the data to its respective servers to faster computation of data and the data gets split into words and those words are filtered to find the keywords and subjects and after the processing, the result or the output is sent back to respective assistant. Few of the most used IVAs are discussed in subsequent subsections.

\section{1-1- Alexa}

Alexa is virtual assistant software that has been developed by Amazon.com. It is based on Artificial Intelligence and it has been mainly written in Node.js and java, python, $\mathrm{C}$ has also been used. Its main purpose was to help the user to get commands in a natural language like humans and also to give output in the same languages which will lead to reducing the human effort. This assistant gets active when the user speaks 'Alexa' then it responds and now the assistant is ready to get commands from the user [1]. This was made to do all the works which other assistant does with a specialty feature that it can also control your smart home devices using your voice. According to the company, it is safe and secured to use and can also help to 
keep you organized. Alexa can be a good competition in the market to the other virtual assistant like google assistant, Siri, and Cortana assistant because of its features. Before the Alexa was only available in smart devices only but it has been offered in smartphones too to compete with other virtual assistants. When you say 'Alexa', "what's the weather today", your speech is recorded in the form of speech of text and which is sent to the main server of amazon's Alexa voice services and there these words are been broken or split into words and the main subject words or nouns are separated from it. After this the result is analyzed and sent back to the device and which has been responded to the user by the Alexa assistant application [2].

\section{1-2- Google Assistant}

Google Assistant is another virtual assistant software application which is developed by Google.com and this assistant is also fully based on Artificial Intelligence and natural language processing and was writ- ten in $\mathrm{C}++$ programming language. This is also like Alexa as it also uses to way communication to get com mands from the user using the It is available on smartphones and other smart home devices [3]. It is available in approximately 31 languages and more are on the coming to way. This assistant gets active when the user speaks 'Okay Google' or in some devices 'Hey Google' then it responds and now the assistant is ready to get commands from the user in any language you want. Like the operating system, the google assistant also works as interface and helps us to interact with the hardware and the software of devices. Google's assistant [4] first records your speech and interpreting the sounds takes a lot of time and computational power so it is sent to Google's servers to be analyzed more efficiently. The important words or nouns or the main subjects words are split from the sentence to carry out the task and after which if the word found is like 'temperature' or 'weather' then it would open the weather app and also the resultant information or the result is sent back to the device is again spoken by the virtual assistant to the user in Figure 1.

\section{1-3-Siri}

Siri is another virtual assistant software application [5] that has been developed by Apple. This virtual assistant is written in Objective-C Programming Language. But Apple did not entirely develop Siri by themselves as It started from an AI initiative in 2003 funded by DARPA (Defense Advanced Research Projects Agency) and run by a Stanford University affiliate, SRI International and Their goal was to make a program that helps military personal with office work and making decisions, resulting in CALO (Cognitive Assistant that Learns and Organizes) that learns from its users and the vast amounts of available data. It was used for organizing and scheduling meetings as well as providing the necessary documents for the participants.

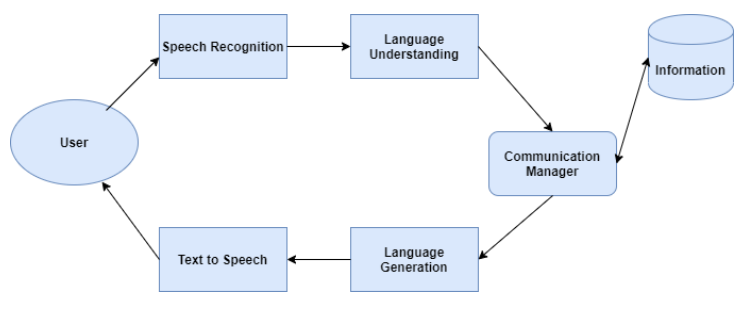

Figure 1. Structure of Virtual Assistants

This application also uses a two-way communication process to get the commands from the user and this is again fully based on Artificial Intelligence and according to the company it is the safest and securest application that manages the data of your device [6]. Siri assistant is available for almost all of the apple de- vices. This assistant uses voice queries and a natural- language user interface to answer questions, make recommendations, and perform actions by delegating re- quests to a set of internet services. Siri comes with both the two voices male and female too. This virtual assistant Siri gets active when the user will speak 'Hey Siri' and then now you are ready to give the command to the Siri or the virtual assistant in your language.

It has come after when iPhone $4 \mathrm{~S}$ has launched [7]. After receiving the request, Siri records the frequencies and the sound waves from the user's voice and translates it into a code. Then it breaks or splits the sentence into words to identify the keyword and the patterns used and then the data gets input into a com- plex algorithm that gets matched and compared to the thousands of combinations of sentences the determine the meaning of the sentence. Once Siri determines what is the request all about or the task which needs to be carried out, then it determines whether the in- formation has to be accessed by the phone's bank or its online servers. After then it can complete its task given by the user and performs the task accordingly to the user with giving the appropriate output.

\section{2- Related Work}

A strategy for directing a call between a guest and an intelligent voice reaction (IVR) framework, the guest utilizing a gadget to lead the call, the gadget arranged to execute a menial helper, the technique involving utilizing the remote helper to lead the call at any rate to some degree by impacting the style of data given to the guest during the call as well as the substance of data went between the gadget and the IVR [8] framework during the call. In intelligent virtual assistant, Intelligent techniques 
are not only used in day to day interaction like play music and open an application but also in business interaction [9]. IVAs advanced from chatbots, programming specialists customized to banter with people through either text or voice. The first chatbot, ELIZA, was created by Joseph Weizenbaum at MIT 16 years after Alan Turing previously proposed his test of man-made consciousness in 1950. ELIZA utilized common language handling to perceive catchphrases in composed info what's more, produce prescripted reactions that to certain clients took after human understanding [10]. Repel, presented in 1972 by a therapist named Kenneth Colby who persuaded various prepared specialists that it was a genuine individual with distrustful schizophrenia. A technique and framework for making a theme-based 3D virtual condition-The imaginative strategy and framework give a 3D virtual condition that incorporates pictures, landscapes, and substances that identify with the subject of conversation in the virtual condition [11]. On the off chance that the virtual "meeting" ought to be suspended or finished, the framework spares the information, conversations that happened during the gathering, and all the landscape and sub-stance identified with the virtual gathering so the gathering can proceed with the entirety of the substance and symbolism set up. A virtual situation format containing this data is stacked and utilized for resulting virtual gatherings talking about a similar theme.

Remote helper [12] is an aid for everybody in this new time of the 21 st century. It has cleared a path for another innovation where we can pose inquiries to the machine and can collaborate with IVAs as individuals do with people. This innovation pulled in the practically entire world from numerous points of view like advanced mobile phones, workstations, PCs, and so forth.

The most well-known use of the iPhone is" Siri" [13] which makes a difference in the end client to convey end client portable with voice and it like- wise reacts to the voice orders of the client. The same kind of utilization is likewise evolved by Google that is "Google Voice Search" which is utilized for Android Phones. Be that as it may, this Application for the most part works with Internet Connections.

Amazon's Echo [14] and its conversational specialist Alexa open energizing open doors for seeing how individuals see and associate with virtual operators. Drawing from client audits of the Echo presented on Amazon.com, this contextual investigation investigates how much client surveys show exemplification of the gadget, friendliness level of associations, factors connected with representation, and effects on client fulfillment.

One of the objectives of Artificial knowledge (AI) [15] is the acknowledgment of common discourse among people and machines. Lately, the exchange frameworks, otherwise called intuitive conversational frameworks are the quickest developing territory in AI. Ongoing advances in programming incorporation and endeavors toward more personalization and set- ting mindfulness have brought nearer the long-standing vision of the omnipresent smart individual collaborator. This has gotten especially striking with regards to cell phones and electronic tablets [16], where com- mon language connections can significantly improve the portable experience. A long way past simply offering more choices as far as UI, this pattern may well attendant in an authentic change in perspective in manmachine correspondence.

Although these advancements appear to be widely received, individuals do not expect to utilize them sometimes. Innovation reception has been read for a long time, and there are many general models in the writing depicting it. Be that as it may, having more models for developing advances upon their high- lights appears to be essential.

In this investigation, we built up a theoretical a model including another framework [17] quality build, i.e., connection quality, which we accept can better depict appropriation of AI- based advances.

A technique for executing a believed application on a believed security zone empowered electronic gadget [18]. The strategy involves receptive to a confided in security subzone not being provisioned on the electronic gadget, creating, by a server, an impermanent trust token, transmitting the brief trust token to the electronic gadget, and contrasting the transitory trust token and a majority of trust tokens put away in the electronic gadget to decide the dependability of the brief trust token.

This paper [19] just highlights communication net- works established over several servers and how they turn to be efficient in their ways. Inbuilt security systems available with IVAs have a significant role in imparting safety and protecting the ethical and civic rights of the internet users. Several threats exist against a security system. The key to successfully entrench a communication network lies in recognizing, extracting, and eliminating such attacks with pre-designed applications that can predict or possibly identify such malware threats at the earliest, thus preventing any damage to the data, devices, or its users.

\section{3- Comparison of different IVAs}

Previously, we have seen how the trust gets build when we connect to computer machines in a network using the IP (Internet Protocol) and Port by socket programming. Now, we will see how the trust gets built when the computer system gets connected with the voice of Human and the command is been executed according to the user by his/her voice as a command. This is a much more efficient and effective way to execute the commands in comparison to the previous one. This reduces the typing error and avoids the chance of getting spelling mistakes while giving the 
command to the user. Previously we used socket programming to build trust among the machine to machine but now will use the natural language processing tool to make it much more efficient and effective. Previously the work was done by encoding and decoding the information in machines at the time of sending and receiving the information to build trust but now this will be reduced and now the voice will work as command and now will focus on the keywords said by the user or the developer to get executed in the machine [20].

All Google Assistant, Siri, and Amazon Alexa are getting smarter day by day with their regular training and their self-capability to learn by using Artificial Intelligence [21].

If we talk about the comparison then the entire voice assistant is almost the same as they have the almost same functionality and the same level of trust but if we go more in detail then we will find some points which make a difference among all these applications [22]. Human Trust is measured in Virtual assistant are based on virtual reality. to measure this Physiological Sensing has been used [23].

\section{3-1-Alexa vs Google Assistant vs Apple's Siri s}

We talk about waking up of these assistants then Amazon's Alexa is better than the Google Assistant as 'the waking word' (the word which will make the assistant active for listening to the user) for the Amazon's Alexa is just one simple word 'Alexa' and Google's Assistant is 'Okay, Google'.

If we talk about data or IQ then Google Assistant will be better than Amazon's Alexa as because Google sells the data to Amazon and its $\mathrm{N}$ number of copies are also sold to several companies by Google, hence we can say that Google will have will more data to serve to us than Amazon . Google has direct access to the indexed information from all searches whereas Alexa has direct access to the indexed information from all purchase's information. The main difference where Google's Assistant can be much better than Amazon's Alexa is that Google has more resources on their AI (Artificial Intelligence) side to serve its Assistant but Amazon is not that much advance but still Amazon has more Applications (skills) that makes it's Alexa currently richer. Google's Assistant is powered with Google Ma- chine learning library and algorithms which get better day by day and providing better voice recognition and better results. After when the human voice is recorded then Google Assistant sends it to the Google server's and Alexa Assistant sends it to its Amazon Alexa Ser- vices server to make computational decisions and its interpretation in Figure 2. If we talk about platforms for working then Apple's Siri is limited to Apple's ecosystem but Google Assistant supports all Android and iOS devices too. Google uses a natural language user interface to respond to queries, and perform actions by delegating the request to a set of services and it is closely integrated with the Google search engine whereas Apple's Siri uses natural language processing to meet searching and operation and it uses it's phone's default search engine to get results. If we talk about data than some- where Google's Assistant can go better than Apple's Siri because Google is the hub of huge data and also sells it to so many other companies whereas Apple uses only it's default browser to search the results. Hence, somewhere Google has more data than Apple to serve its users.

Both the assistant works similar like both can play song, radio, answer your questions and provide information regarding the traffic of your area and weather but somewhere Google Assistant becomes better as it excels in terms of handling search-based web queries because searching is Google's main functionality sown in Table 1 and it becomes more natural to use whereas Siri is the oldest and one of the most used digital assistants out there as it was launched at the time when iPhone $4 \mathrm{~S}$ was launched and it's most common uses web browsing and dictation. If we talk in terms of integration, then Google's Assistant becomes better as it works with more than 5,000 smart home devices from than 100s of brands whereas Siri is just limited to Apple's Ecosystem only with a very limited range of devices supported in an individual brand.

Table 1: Comparison of different application of IVA

\begin{tabular}{|l|c|l|c|}
\hline Music & Alexa & Google & Siri \\
\hline Amazon Music & Yes & No & No \\
\hline Apple Music & Yes & No & Yes \\
\hline Deezer & Yes & Yes & No \\
\hline Google Play Music & No & Yes & No \\
\hline iHeartRadio & Yes & No & No \\
\hline Pandora & Yes & Yes & No \\
\hline SiriusXM & Yes & No & No \\
\hline Spotify & Yes & Yes & No \\
\hline TuneIn & Yes & No & No \\
\hline Tidal & Yes & No & No \\
\hline YouTube Music & No & Yes & No \\
\hline
\end{tabular}

\section{4- Trust issues in Intelligent Virtual Assistant}

Before exploring cybersecurity issues [6], we need to list down all the applications of IoT. Corporations, Governments, and people are the three-broad class of IoT stakeholders. Corporations provide IoT devices, Governments are responsible for the regulation of IoT devices, and people are the end-users. Based on the IoT device usage point, these can be divided into A. Personal / Household IoT Devices A smart home is probably the first 
thing that comes to anyone's mind when we talk about IoT devices. Home Lights, AC, TV, speakers, washing machine, refrigerator, oven, etc. are some examples of Household IoT devices. Smart- watch is an example of a personal IoT device.

Public IoT Devices After a smart home, a smart city is also getting popular. Smart cities, based on IoT devices, will be energy efficient, economical, environmentally friendly, more secure, and better administered.

Transportation, economy, health, environment, security, and administration are broad application classes for public IoT devices. Using GPS [10] and public transportation card, we can better monitor bus timing and peak in public headcount. We can give real-time bus timing to everyone using GPS tracking [7]. Extra buses can be arranged in peak hours after the analysis of transportation card data. Traffic can also be diverted between different routes using GPS tracking.

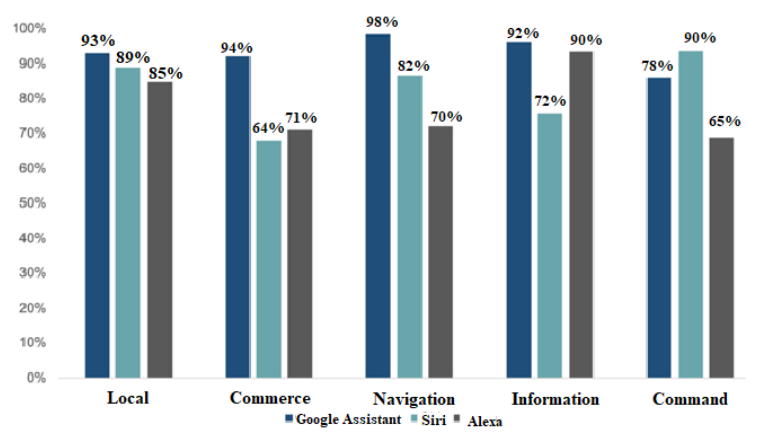

Figure 2. Comparison of Google Assistant, Siri and Alexa

Tourism can be benefitted by sending push- notifications of nearby attractions, restaurants, malls, etc. to tourists in the city. Better infrastructure to selective locations can be provided after an analysis of tourist's footfall data. The emergency button in our watch or smartphone can provide a timely response from the hospital [9], fire department, police, etc. Automate health devices can send an alert to your doctor directly if an abnormal health condition is observed like high sugar level, high blood pressure, etc. The environment can be benefited largely by using different sensors for water pipeline leakage detection and air population-level detection. Real-time responses can be activated to tackle these. Sensors in gas-pipeline and smoke detectors can send timely alerts even before any mishappening. Drones and traffic cameras can identify a report any security issues. Overall, we will live in a more secure city. Using biometric [8] and Aadhar like services, the administration can be more efficient and faster. Services can be delivered directly on smart- phones. Apart from these, innovations are happening daily in public IoT applications.

The recent convergence of multiple technologies and IoT or Internet of Things (A system of interrelated computer devices or $\mathrm{M} 2 \mathrm{M}$ ) has become the foundation for the concept of 'Smart Homes' which include home security systems, smart lighting, thermostats, etc. these can then be controlled using devices which are considered as userfriendly access points such as smartphones and speakers (Artificial Intelligence-based software).

Trust in such technologies enables users to make their everyday life more time-friendly, pragmatic, and sorted efficiently. Essentially that is the basis of the popularity of AI software like Google Home, Amazon's Alexa and Echo, Apple's- watch, home-pod, home-kit, etc. Such facilities are extremely beneficial during emergencies. In cases of medical emergencies, a person's medical record may be shared over multiple interfaces to make it accessible to the doctors treating the person. Trust and better communication across all devices sharing a network make situations like this possible. Of course, there is the fear that this trust may be misused to procure data by unauthorized personnel. As such threats do exist in the real world, every soft- ware developing company, by default, builds security firewalls to protect the user data and grant exclusive data-sharing privileges to devices that the user trusts. Trusted devices also assist people suffering from dis- abilities, injuries, and even elderly people. Cochlear implants, pacemakers, other specialized implants, voice control navigation, assistive touch, etc. all help in flexibly accommodating the needs of such individuals.

So now the question arises that how can we compute trust in a model where we have no intuition about how will it work internally? A reliable basis for trust could be testing. But to 'test' a model we need to list down our expectations from that model. Firstly, in that case a complete specification of the model itself would be generated. But to methodize our expectations is not an easy task because the concept of 'fairness', cannot be given or presented through any mathematical algorithm. Trust in a machine could also be entrusted through experience.

The experience a ma- chine already incorporates in any user could be a re- liable basis to generate the trust of that user in that machine or program. Another method to compute trust can be feature. The amount and level of features a machine or a program promises to a user is directly dependent on the number of users generating their trusts on that machine. But again, testing the machine first and then going for the features and blindfold trusting them would be more precautionary. 


\section{5- Computational Trust Model for IVA}

The idea or plan behind "trust management" is to develop a relationship or bond based on trust worthiness among all the nodes or among users and nodes those are involved in computational trust model. Here we consider nodes as different IVAs. As it is essential to understand without the trust among all the participating nodes and users the communication cannot establish. While on the other hand, maintaining the trust relationship between the systems, objects etc. It is vital to make sure that the complete system is more structured in terms of security. Naive Bayes, more technically referred to as the Posterior Probability, updates the prior belief of an event given new information. The result is the probability of the class occurring given the new data. Naive Bayes theorem is one of the simplest probabilistic classifiers, based on the Bayes theorem with strong (naive) independence assumptions. Naive Bayes is used to predict class membership probabilities, i.e. the probability that a given sample belongs to a category.

$$
f\left(x \mid \mu, \sigma^{2}\right)=\frac{1}{\sqrt{2 \pi \sigma^{2}}} e^{\frac{-(x-\mu)^{2}}{2 \sigma^{2}}}
$$

where ' $\mu$ ' and ' $\sigma 2$ are the average and the variance respectively of the values in $\mathrm{X}$.

As Naïve Bayes theorem has different characteristics including simplest probabilistic classifiers, able to handles both continuous and discrete data, scalability in term of number of predictions and data points and fast convergence to make real time predictions. These are reasons to choose Naïve Bayes theorem to compute trust and furthers evaluate probability of using trusted IVA devices.

Prior probability, in Bayesian statistical inference, is the probability of an event before new data is collected. This is the best rational assessment of the probability of an outcome based on the current knowledge before an experiment is performed. To calculate Bayes' probability following formula is used

$\mathrm{P}(\mathrm{A} \mid \mathrm{B})=[\mathrm{P}(\mathrm{B} \mid \mathrm{A}) * \mathrm{P}(\mathrm{A})] / \mathrm{P}(\mathrm{B})$, where: $\mathrm{A}$ and $\mathrm{B}$ are certain events. $\mathrm{P}(\mathrm{A})$ is the prior probability of event $\mathrm{A}$ occurring. Three different classes of prior probability $\mathrm{p}<.025, \mathrm{p}<.05, \mathrm{p}<.001$ are used.

\section{6- Implementation, Result and Analysis}

We have implemented Naïve Bayes classifier in Python language. To perform result analysis, different IVA users and non-users $(\mathrm{N}=1000)$ are surveyed. This survey is performed to understand and analyze the barriers and motivations to adopting IVAs and how users are concerned about data privacy and trust with respect to organizational compliances and social contract related to IVA data and how these concerns have affected the acceptance and use of IVAs.

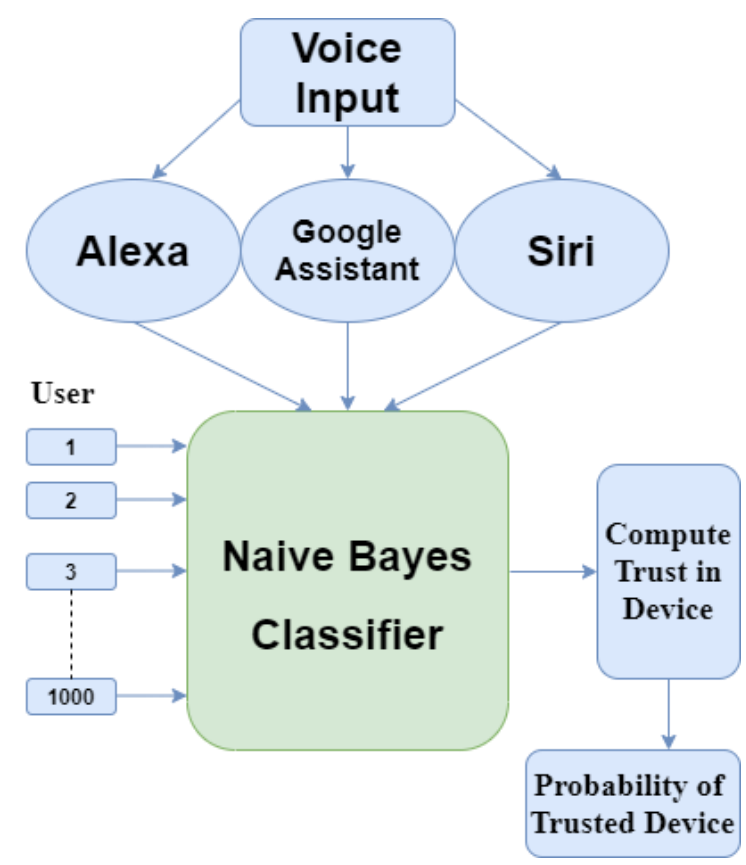

Figure 3. Computational Trust Model

We have used Naïve Byes Classifier to compute trust in IVA devices and further evaluate probability of using different trusted IVA devices.

\section{6-1- Research Question 1: Find out key Reasons to Use or Non-use of IVAs}

\section{Key reasons to using IVAs-}

Out of 1000 respondents 652 respondents who had/have ever utilized a Phone IVA-including the individuals who utilized it and the individuals who had utilized it beforewe asked them to recognize for what valid reason they utilized it. The overview incorporated a rundown of 11 potential reasons, (in addition to an open-finished alternative), and respondents could choose various reactions. The most mainstream purposes behind utilizing a Phone IVA were: (1) posing genuine inquiries (82\%); (2) getting bearings/area of a spot (67\%); (3) asking senseless/clever inquiries only for chuckles $(62 \%)$; (4) directing an instant message or email (58\%); (5) set- ting a clock (49\%). Extra reactions that gathered fewer votes included asking counsel, asking wellbeing explicit inquiries and home robotization. For the 380 respondents 
who announced possessing a Google Home or Amazon Alexa, we approached them to list their inspirations for buying the gadget. Almost half (48\%) said they had gotten the IVA as a blessing; others said they had bought the gadget essentially to control home gadgets (17\%), to clear something up or for no particular reason (18\%), to stream music (11\%), and to have sans hands access to online data $(15 \%)$.

Key barriers to not using IVAs- For the 457 respondents who said they didn't utilize a Phone IVA-including the individuals who had never utilized it and the individuals who had deactivated IVA highlights on their telephonewe requested that they rate factors that may have assumed a job in their choice. The variables regularly referred to by these respondents reflected worries about utility, privacy and protection.

Table 2. Probability of use the IVAs on behalf of trust and privacy

\begin{tabular}{|l|l|l|}
\hline \multicolumn{3}{|c|}{ Probability of use the IVAs on behalf of trust and privacy } \\
\hline \multicolumn{3}{|c|}{ Odds Ratio for different probabilities } \\
\hline Classification & $\begin{array}{c}\text { Case 1 } \\
\text { Phone IVA }\end{array}$ & $\begin{array}{c}\text { Case2 } \\
\text { Home IVA }\end{array}$ \\
\hline Gender & $-.24(.80)$ & $.00(1.02)$ \\
\hline Age Group (Young/Adult) & $.03(1.01)^{* * *}$ & $-.00(.98)$ \\
\hline Working/not working & $-.00(1.00)$ & $.10(1.14)^{* *}$ \\
\hline Digital Native/Digital Migrant & $.57(1.76)^{* * *}$ & $.23(1.22)^{*}$ \\
\hline $\begin{array}{l}\text { Types (Siri, Alexa and Google } \\
\text { Assistance) }\end{array}$ & $.43(1.54)^{* * *}$ & $-.03(.94)$ \\
\hline Trust based on privacy and security & \\
\hline Issues related to general privacy & $-.17(.84)^{*}$ & $.02(1.00)$ \\
\hline Issues related to smart phone privacy & $.03(1.01)$ & .34 \\
\hline Concerns/Issues related to IVA data & $-.08(.92)$ & $(1.44))^{* *}$ \\
\hline Data confidence Concerns/Issues & $.34(1.34)^{* * *}$ & $.26(1.32)^{*}$ \\
\hline Type of IVA used & $.52(1.52)^{* * *}$ & - \\
\hline Home IVA & - & .54 \\
\hline Phone IVA & & $(1.72)^{* *}$ \\
\hline Apply equation 1( Naïve Bayes & $2=140.0$, & $2=114.98$, \\
Classifier) & $=11 * * *$ & $=11 * * *$ \\
\hline Google Assistance & 0.7 & 0.65 \\
\hline Siri & 0.83 & 0.81 \\
\hline Alexa $<.05, * * * p<.001$ & 0.79 \\
\hline \multicolumn{3}{|c|}{0.76} \\
\hline
\end{tabular}

\section{6-2- Research Question 2: Predicting Adoption and Use of IVAs based on Trust and Privacy}

Binary logistic regression models are used to classify user and non-users of IVAs. Three major IVAs Siri, Alexa and Google Assistant are evaluated by users. Table 1 summarizes the "Probability of use the IVAs on behalf of trust and privacy".
To find this probability different variables and parameters including background and demographic variables, privacy and security concerns, and experience with three types of IVAs (Siri, Alexa and Google Assistance). Results from Naïve Byes based Trust management model has indicated that general privacy concerns are major parameter. Three different classes of probability $\mathrm{p}<.025, \mathrm{p}<.05, \mathrm{p}<.001$ are used. After applying Naïve Bayes Classifier, we have find out that Siri has higher probability of use as users and nonusers have shown more confidence in iPhone based Siri due to high level of security and privacy which enhanced their trust.

\section{7- Conclusions}

Besides the IoT perspective, Trust management must provide uniform decisions. Despite all the hard work performed by various researchers and scientists on this aspect to provide a reliable uniform trust managing scheme, it will remain a broad and complex field that will always remain open for any further researches and proposals. There seem to be around 200 billion devices and sensors by 2020 which will be interconnected in a way to make things and life better for us. Thus, their importance is immense in the coming future. Due to their rising importance, it is also necessary to limit the security threats to which the people and the government could become vulnerable. The more connected the world becomes, the more the risks arise. To evaluate trust, we have surveyed 1000 respondents. While we found a way to arrive at a differing set of respondents through our inspecting technique, we were obliged to college representatives, prompting a profoundly taught test. Moreover, results depended on a onetime study and hence give a preview of a specific second in time. The outcomes from this investigation uncover a perplexing picture of IVA clients and non-clients.

\section{Acknowledgments}

Authors of this research paper have expressed their sincere gratitude to the administration of Amity Institute of Information Technology (AIIT), Amity University Uttar Pradesh (AUUP), India for providing the academic environment to pursue research activities. 


\section{References}

[1] J. Caminha, A. Perkusich, and M. Perkusich, "A smart trust management method to detect on-off attacks in the internet of things," Security Commun. Netw., vol. 2018, pp. 1-10, Apr. 2018.

[2] A.V. Singh, V. Juyal and R.Saggar, "Trust based Intelligent Routing Algorithm for Delay Tolerant Network using Artificial Neural Network" in Wireless Networks (WINE), US:Springer Publication, vol. 22, no. 135, pp. 1-10, Jan. 2016.

[3] G. D'Angelo, S. Rampone and F. Palmieri, "An Artificial Intelligence based Trust Model for Pervasive Computing", Proceedings on 10th International Conference on P2P Parallel Grid Cloud and Internet Computing 3PGCIC 2015 art. no. 7424653, pp. 701-706, 2015.

[4] K. Siau and W. Wang, Building Trust in Artificial Intelligence, Machine Learning, and Robotics, Cutter Business Technology Journal, Vol. 31, No. 2, pp. 47-53, 2018.

[5] I.-R. Chen, F. Bao and J. Guo, "Trust-based service management for social Internet of Things systems", IEEE Trans. Depend. Secure Comput., vol. 13, no. 6, pp. 684-696, Nov./Dec. 2016.

[6] S. M. Sony and S. B. Sasi, "On-Off attack management based on trust", In 2016 Online International Conference on Green Engineering and Technologies (IC-GET), pp. 1-4, November, 2016.

[7] F. Jiang et al., "Deep learning based multi-channel intelligent attack detection for data security", IEEE Trans. Sustain. Comput., [online] Available: https://ieeexplore.ieee.org/document/8259310/.

[8] J. Jiang, G. Han, C. Zhu, S. Chan and J. J. P. C. Rodrigues, "A trust cloud model for underwater wireless sensor networks", IEEE Commun. Mag., vol. 55, no. 3, pp. 110-116, Mar. 2017.

[9] Kot, Mateusz Tomasz, and Grzegorz Leszczyński. "The concept of intelligent agent in business interactions: is virtual assistant an actor or a boundary object?." Journal of Business \& Industrial Marketing (2020).

[10] F. Fei, S. Li, H. Dai, C. Hu and W. Dou, "A K-anonymity based schema for location privacy preservation", IEEE Trans. Sustainable Comput.2017.

[11] S. Talari et al., "A review of smart cities based on the Internet of Things concept", Energies, vol. 10, no. 4, pp. 421, 2017.

[12] F. Boustanifar and Z. Movahedi, "A trust-based offloading for mobile M2M communications", Proc. Int. IEEE Conf. Ubiquitous Intell. Comput., pp. 1139-1143, 2016.

[13] Gartner Says Worldwide Spending on VPA-Enabled Wireless Speakers Will Top $1 \$ 2$ Billion by 2020, Gartner, Oct. 2016, [online] Available: www.gartner.com/newsroom/id/3464317.

[14] A. Liptak, Amazon's Alexa Started Ordering People Dollhouses after Hearing Its Name on TV, The Verge, Jan. 2017, [online] Available: www.theverge.com/2017/1/7/14200210/amazon-alexa-technews-anchor-order-dollhouse.

[15] K. York, Dyn Statement on 10/21/2016 DDoS Attack, [online] Available: dyn.com/blog/dyn-statement-on10212016-ddos-attack, Oct. 2016.
[16] Yuting Liao, Jessica Vitak, Priya Kumar, Michael Zimmer, and Katherine Kritikos. Understanding the Role of Privacy and Trust in Intelligent Personal Assistant Adoption. In Information in Contemporary Society, Natalie Greene Taylor, Caitlin Christian-Lamb, Michelle H. Martin and Bonnie Nardi (eds.). Springer International Publishing, Cham, 102--113. https://doi.org/10.1007/978--3-030--15742$-5 \_9,2019$.

[17] Chung, Hyunji, Michaela Iorga, Jeffrey Voas, and Sangjin Lee. Alexa, Can I Trust You? Computer 50: 100, 2017.

[18] A. S. Tulshan and S. N. Dhage, "Survey on virtual assistant: Google assistant Siri Cortana Alexa", Proc. Int. Symp. Signal Process. Intell. Recognit. Syst., pp. 190-201, 2018.

[19] Kshama V. Kulhalli, "Personal Assistant with Voice Recognition Intelligence", ISSN 0974-3154 Volume 10, Number 1, 2017.

[20] Amanda Purington, Jessie G. Taft, Shruti Sannon, Natalya N. Bazarova, and Samuel Hardman Taylor. 2017. "Alexa is my new BFF": Social Roles, User Satisfaction, and Personification of the Amazon Echo. In Proceedings of the CHI Conference Extended Abstracts on Human Factors in Computing Systems (CHI EA '17). ACM, New York, NY, USA, 2853-2859, 2017.

[21] V. Këpuska and G. Bohouta, "Next-Generation of Virtual Personal Assistants (Microsoft Cortana, Apple Siri, Amazon Alexa and Google Home)," in 2018 IEEE 8th Annual Computing and Communication Workshop and Conference (CCWC), pp. 99-103, 2018.

[22] J. R. Bellegarda, "Spoken language understanding for natural interaction: The Siri experience" in Natural Interaction with Robots Knowbots and Smartphones, New York:Springer-Verlag, pp. 3-14, 2014.

[23] Kunal Gupta, Ryo Hajika, Yun Suen Pai, Andreas Duenser, Martin Lochner, and Mark Billinghurst. "Measuring Human Trust in a Virtual Assistant using Physiological Sensing in Virtual Reality." In 2020 IEEE Conference on Virtual Reality and 3D User Interfaces (VR), pp. 756-765. IEEE, 2020.

Babu Kumar is research scholar in Amity University, Noida, U.P, India. He has received his MTech. in Computer science from National Institute of Technology, Uttarakhand, India. His area of research is Intelligent Trust Computational Modeling for Machine to Machine Communication. He has two research publications in area of Trust Computational published in National/International conferences. He has consistently maintained percentage above distinction throughout his academic career.

Ajay Vikram Singh has received his Ph.D. in Computer Science from Jamia Hamdard University, Delhi, India. He has completed his Master's in Computer Application from IIT Roorkee, India. His area of research are Ad hoc Networks, Internet of Things and Soft Computing Techniques. He has 17 years of teaching and research experience. Currently he is working as associate professor in Amity Institute of Information Technology (AlIT), Amity University Uttar Pradesh, Noida, India. 
Parul Agarwal has received her Ph.D. in Computer Science from Jamia Hamdard University, Delhi, India. She has completed her master's in computer application from IIT Roorkee, India. Her area of specialization includes Fuzzy Data Mining, Cloud Computing, and Soft Computing. Her interest includes Sustainable Computing and its applications in Agriculture, transportation, Smart Cities and health care. She has published several papers in reputed and $\mathrm{SCl}$, Scopus and Elsevier indexed journals and many book chapters published by CRC, Springer, IGI-Global are to her credit. She is working as associate professor in Department of Computer Science, Jamia Hamdard University, New Delhi, India. 